\title{
The non-invasive serum biomarker soluble AxI accurately detects advanced liver fibrosis and cirrhosis
}

\author{
Katharina Staufer ${ }^{1,2}$, Mirko Dengler ${ }^{3}$, Heidemarie Huber ${ }^{3}$, Rodrig Marculescu ${ }^{4}$, Rudolf Stauber ${ }^{5}$, Carolin Lackner ${ }^{6}$, Hans-Peter Dienes ${ }^{7}$, \\ Danijel Kivaranovic ${ }^{8}$, Christian Schachner ${ }^{2}$, Markus Zeitlinger ${ }^{9}$, Beatrix Wulkersdorfer ${ }^{9}$, Peter Rauch ${ }^{10}$, Gerhard Prager ${ }^{11}$, \\ Michael Trauner ${ }^{2}$ and Wolfgang Mikulits*,3
}

Soluble Axl (sAxl) was recently shown to be strongly released into the blood during liver fibrogenesis and hepatocellular carcinoma suggesting sAxl as a biomarker of liver diseases. In this study we are the first to evaluate sAxl in human serum in comparison to Enhanced Liver Fibrosis (ELF) test and transient elastography (TE; Fibroscan) for its value to detect significant $(F \geq 2)$, advanced fibrosis $(F \geq 3)$, and cirrhosis ( $F 4)$ in different liver disease etiologies and healthy controls. To properly determine the diagnostic accuracy of $\mathrm{sAxl}$, a test cohort as well as a validation cohort was employed using liver biopsy as a reference method. Most notably, sAxl was confirmed to be an accurate biomarker of liver fibrosis and cirrhosis. Its accuracy was increased, if total serum albumin was added to build a sAxl/albumin ratio. Thereby an AUC of $0.763,0.776,0.826$, and 0.832 was achieved corresponding to histological fibrosis stages $F \geq 2, F \geq 3$, F4 with liver biopsy as a reference method, and cirrhosis according to imaging techniques, respectively. With a cut-off of 1.29 , a sensitivity, specificity, PPV, and NPV of $78.5 \%, 80.1 \%, 44 \%, 94.9 \%$ for the detection of cirrhosis was achieved. In comparison, ELF test and TE showed an AUC of 0.910, and 0.934, respectively, for the detection of cirrhosis. However, performance of TE was not possible in $14.4 \%$ of patients and both, ELFTM test and TE bear the disadvantage of high costs. In conclusion, the sAxl/albumin ratio is suggested as an accurate biomarker of liver fibrosis and cirrhosis. Due to its easy applicability and low costs it is suitable as screening parameter for significant to advanced liver fibrosis and cirrhosis, especially if TE is not available or not applicable.

Cell Death and Disease (2017) 8, e3135; doi:10.1038/cddis.2017.554; published online 26 October 2017

Detection of advanced liver fibrosis and cirrhosis is crucial to support therapeutic decisions, determine surveillance intervals, and predict clinical outcome. ${ }^{1-3}$ Liver fibrosis has been shown to be the most significant predictor of liver-related mortality in several liver diseases. ${ }^{4,5}$ A variety of serum-based biomarker panels, frequently combined with clinical parameters, such as body mass index (BMI), age, or the presence of diabetes, have been investigated in comparison to liver biopsy as a non-invasive, lower-cost alternative. All of these markers, like APR Index (APRI), ${ }^{6,7}$ Enhanced Liver Fibrosis (ELF) test, ${ }^{8,9}$ Fibrosis 4 (FIB4)-Index, ${ }^{10}$ Non-alcoholic fatty liver disease (NAFLD) fibrosis score ${ }^{11}$ Cirrhosis Probability in Hepatitis C (Lok) index ${ }^{12}$ or FibroTest, ${ }^{13,14}$ were analysed in various liver diseases, mostly associated with non-alcoholic fatty liver disease (NAFLD) and viral hepatitis showing areas under the receiver operating curve (AUROC) of 0.67 to 0.94 for the detection of significant fibrosis $(F)(F \geq 2)$, or advanced fibrosis $(F \geq 3)$, respectively. ${ }^{3}$ Furthermore, transient elastography (TE) has been extensively studied for its applicability in routine clinical use. In this context, Fibroscan (Echosens,
Paris, France), Acoustic Radiation Forced Impulse (ARFI; Siemens Healthcare Diagnostics Inc., NY, USA), and Supersonic Shear Imaging (SSI) have been evaluated mainly in NAFLD, ${ }^{15,16}$ viral hepatitis ${ }^{17}$ and alcoholic liver disease (ALD). ${ }^{18}$ Limitations of these techniques are identified for specific patient populations such as patients with acute hepatitis, congestive heart failure, obesity, ascites or patients after abdominal surgery possibly leading to intraabdominal adhesions and are associated with increased expenses. ${ }^{3}$ The optimal non-invasive biomarker for liver fibrosis includes high specificity and sensitivity, is independent of liver disease etiology and body mass index (BMI), is easily accessible and cost - effective. . $^{3,19}$

Enhanced levels of soluble Axl (sAxl) in combination with the ligand of the Axl receptor, Gas6, have been detected in liver cirrhosis and suggested as a diagnostic tool. ${ }^{20-22}$ Recently, we reported that sAxI is an accurate biomarker for advanced fibrosis, cirrhosis and hepatocellular carcinoma (HCC) showing an AUC of 0.918 for the detection of biopsyproven advanced fibrosis ( $\mathrm{F} \geq 3)$, and an AUC 0.935 for the

\footnotetext{
${ }^{1}$ Division of Transplantation, Department of Surgery, Medical University of Vienna, Vienna, Austria; ${ }^{2}$ Division of Gastroenterology and Hepatology, Department of Internal Medicine III, Medical University of Vienna, Vienna, Austria; ${ }^{3}$ Department of Internal Medicine I, Institute of Cancer Research, Comprehensive Cancer Center Vienna, Medical University of Vienna, Vienna, Austria; ${ }^{4}$ Department of Laboratory Medicine, Medical University of Vienna, Vienna, Austria; ${ }^{5}$ Division of Gastroenterology and Hepatology, Department of Internal Medicine, Medical University of Graz, Graz, Austria; ${ }^{6}$ Institute of Pathology, Medical University of Graz, Graz, Austria; ${ }^{7}$ Clinical Institute of Pathology, Medical University of Vienna, Vienna, Austria; ${ }^{8}$ Department of Statistics and Operations Research, University of Vienna, Vienna, Austria; ${ }^{9}$ Department of Clinical Pharmacology, Medical University of Vienna, Vienna, Austria; ${ }^{10}$ Candor Bioscience GmbH, Wangen im Allgäu, Germany and ${ }^{11}$ Division of General Surgery, Department of Surgery, Medical University of Vienna, Vienna, Austria

*Corresponding author: W Mikulits, Division: Institute of Cancer Research, Department of Medicine I, Medical University of Vienna, Borschkegasse 8a, Vienna 1090, Austria. Tel: +43 140160 57527; Fax: +43 140160 957506; E-mail: wolfgang.mikulits@meduniwien.ac.at

Received 02.6.17; revised 28.7.17; accepted 13.9.17; Edited by M Piacentini
} 
Table 1 Patient characteristics

\begin{tabular}{|c|c|c|c|c|}
\hline & $\begin{array}{c}\text { All patients } \\
N=392\end{array}$ & $\begin{array}{c}\text { Test cohort } \\
N=307\end{array}$ & $\begin{array}{l}\text { Validation cohort } \\
\qquad N=85\end{array}$ & $P$-value \\
\hline $\begin{array}{l}\text { Age (y); median (IQR) } \\
\text { Male sex; \% ( } n) \\
\text { BMI; median (IQR) }\end{array}$ & $\begin{array}{l}50.8(38.3 ; 59.5) \\
55.0(216) \\
26.5(23.7 ; 31.3)\end{array}$ & $\begin{array}{l}50.4(37.1 ; 59.8) \\
56.7(174) \\
26.6(23.7 ; 33.3)\end{array}$ & $\begin{array}{l}52.0(43.0 ; 58.5) \\
\quad 49.4(42) \\
26.0(23.5 ; 28.5)\end{array}$ & $\begin{array}{l}0.207 \\
0.233 \\
0.074\end{array}$ \\
\hline $\begin{array}{l}\text { Liver disease etiology; \% (n) } \\
\text { NAFLD } \\
\text { Viral hepatitis } \\
\text { AlH/CLD/Overlap } \\
\text { PBC/PSC } \\
\text { AlH/Overlap } \\
\text { DILI } \\
\text { ALD } \\
\text { Cryptogenic }\end{array}$ & $\begin{array}{c}43.6(171) \\
21.9(86) \\
18.1(71) \\
8.6(34) \\
9.4(37) \\
2.0(8) \\
4.1(16) \\
2.3(9)\end{array}$ & $\begin{array}{c}45.3(139) \\
15.6(48) \\
19.2(59) \\
9.8(30) \\
9.4(29) \\
2.0(6) \\
5.2(16) \\
2.6(8)\end{array}$ & $\begin{array}{c}37.6(32) \\
44.7(38) \\
14.1(12) \\
4.7(4) \\
9.4(8) \\
2.4(2) \\
0.0(0) \\
1.2(1)\end{array}$ & $\begin{array}{r}0.040 \\
<0.001 \\
0.141 \\
0.349 \\
1.000 \\
1.000 \\
0.016 \\
0.692\end{array}$ \\
\hline $\begin{array}{l}\text { Healthy controls } \\
\text { Cirrhosis according to imaging; \% (n) } \\
\text { US only } \\
\text { US + CT } \\
\text { US + MRI } \\
\text { US + CT + MRI }\end{array}$ & $\begin{aligned} 7.9(31) \\
16.6(65) \\
63.1(41) \\
15.4(10) \\
13.8(9) \\
7.7(5)\end{aligned}$ & $\begin{array}{l}10.1(31) \\
16.9(52) \\
53.8(28) \\
19.2(10) \\
17.3(9) \\
9.6(5)\end{array}$ & $\begin{aligned} & 0.0(0) \\
& 15.3(13) \\
& 100(13) \\
& 0(0) \\
& 0(0) \\
& 0(0)\end{aligned}$ & $\begin{array}{l}\text { n.d. } \\
0.718\end{array}$ \\
\hline $\begin{array}{l}\text { Liver biopsy; \% (n) } \\
\text { sAxl; \% }(n) \\
\text { ELF test, \% }(n) \\
\text { Fibroscan; \% }(n)\end{array}$ & $\begin{array}{l}79.3(311) \\
100(392) \\
84.7(332) \\
33.4(131)\end{array}$ & $\begin{array}{l}73.6(226) \\
100(307) \\
83.4(256) \\
21.2(65)\end{array}$ & $\begin{array}{r}100(85) \\
100(85) \\
89.4(76) \\
77.6(66)\end{array}$ & $\begin{array}{l}\text { n.d. } \\
\text { n.d. } \\
\text { n.d. } \\
\text { n.d. }\end{array}$ \\
\hline
\end{tabular}

Abbreviations: AlH, autoimmune hepatitis; ALD, alcoholic liver disease; CT, computed tomography; CLD, cholestatic liver disease; DILI, drug-induced liver injury; IQR, interquartile range; MRI, magnetic resonance imaging; n.d., not determined; NAFLD, non-alcoholic fatty liver disease; PBC, primary biliary cholangitis; PSC, primary sclerosing cholangitis; US, ultrasound

detection of F4 as compared to healthy controls. ${ }^{23}$ In the present study, we re-evaluated $\mathrm{sAxl}$ as a biomarker of liver fibrosis in comparison to ELF ${ }^{\mathrm{TM}}$ test and TE using liver biopsy as a reference method in a test and a validation cohort.

\section{Results}

Study population. In total, 392 (median age: 50.8y; male sex: $55 \%$ ) patients were included. Of these, 361 patients showed chronic liver diseases, i.e. NAFLD, viral hepatitis (either chronic hepatitis $B$ or $C$ ), autoimmune hepatitis $(A I H$, cholestatic liver disease (CLD, primary sclerosing cholangitis [PSC] and primary biliary cholangitis [PBC]), or overlap syndrome, ALD, drug-induced liver injury (DILI), and cryptogenic cirrhosis (Table 1). The test cohort, which consisted of 276 patients with chronic liver disease and 31 healthy controls (Medical University of Vienna), was compared to a validation cohort (Medical University of Graz) of 85 patients. Both cohorts did not significantly differ in terms of age, sex, and BMI distribution. None of the patients suffered from significant heart failure. In $4.1 \%$ of patients (15/361), chronic kidney disease (CKD) was present. None of the patients had advanced stages of CKD (4 or 5). Detailed patient characteristics are displayed in Table 1.

Histopathological results were available in 311 of 392 study participants. F2, F3 and F4 were present in 20.9\% $(n=65)$, $10.3 \%(n=32)$ and $20.3 \%(n=63)$ of patients, respectively (Table 2a). According to imaging techniques, cirrhosis was present in $16.6 \%(n=65)$ of patients (Table 1$)$ (confirmed by liver biopsy in 63/65, no liver biopsy available in $2 / 65$, no cirrhosis present in 327 patients). The distribution of fibrosis grades among the different liver disease etiologies, as
Table 2a Fibrosis grades based on liver biopsy

\begin{tabular}{ccccc}
\hline & $\begin{array}{c}\text { All patients } \\
\boldsymbol{N}=\mathbf{3 1 1 / 3 9 2}\end{array}$ & $\begin{array}{c}\text { Test cohort } \\
\mathbf{N}=\mathbf{2 2 6 / 3 0 7}\end{array}$ & $\begin{array}{c}\text { Validation } \\
\text { cohort } \\
\mathbf{N = 8 5 / 8 5}\end{array}$ & P-value \\
\hline \multicolumn{2}{l}{ Fibrosis stage; \%(n) } & & & \\
0 & $21.9(68)$ & $20.4(46)$ & $25.9(22)$ & 0.155 \\
1 & $26.7(83)$ & $26.5(60)$ & $27.1(23)$ & 0.987 \\
2 & $20.9(65)$ & $21.7(49)$ & $18.8(16)$ & 0.517 \\
3 & $10.3(32)$ & $9.3(21)$ & $12.9(11)$ & 0.379 \\
4 & $20.3(63)$ & $22.1(50)$ & $15.3(13)$ & 0.155 \\
\hline
\end{tabular}

Liver biopsy was available in 311 of 392 patients

Table $\mathbf{2 b}$ Distribution of fibrosis grades stratified according to liver disease etiology $(n=311)$

\begin{tabular}{lcccc}
\hline $\begin{array}{l}\text { Liver disease etiology; } \\
\%(\boldsymbol{n})\end{array}$ & $\mathbf{F 0 - 1}$ & $\mathbf{F 2}$ & $\mathbf{F 3}$ & F4 \\
\hline NAFLD & $62.0(93)$ & $13.3(20)$ & $11.3(17)$ & $13.3(20)$ \\
Viral hepatitis & $36.0(31)$ & $26.7(23)$ & $12.8(11)$ & $24.4(21)$ \\
AlH/CLD/Overlap & $18.6(8)$ & $46.5(20)$ & $9.3(4)$ & $25.6(11)$ \\
DILI & $87.5(7)$ & $12.5(1)$ & $0.0(0)$ & $0.0(0)$ \\
ALD & $0.0(0)$ & $10.0(1)$ & $0.0(0)$ & $90.0(9)$ \\
Cryptogenic & $77.8(7)$ & $0.0(0)$ & $0.0(0)$ & $22.2(2)$ \\
\hline
\end{tabular}

determined by liver biopsy, is given in Table $2 \mathrm{~b}$. Further, all results of liver function tests are displayed in Table 2c.

sAxI levels depend on stage of liver fibrosis. sAxI levels according to liver disease etiology are presented in Table 3. Notably, sAxI levels in patients with AIH/CLD were significantly higher in the validation cohort. This was explained by 
Table 2c Laboratory parameters of patients with chronic liver diseases

\begin{tabular}{|c|c|c|c|c|}
\hline Laboratory parameter & $\begin{array}{l}\text { All patients } \\
n=360 / 392\end{array}$ & $\begin{array}{l}\text { Test cohort } \\
n=275 / 307\end{array}$ & $\begin{array}{l}\text { Validation cohort } \\
\qquad n=85 / 85\end{array}$ & $P$-value \\
\hline AST (U/ml) median (IQR) & $42.5(29.0 ; 68.0)$ & $39.0(27.0 ; 64.0)$ & $53.0(34.0 ; 75.0)$ & 0.004 \\
\hline ALT (U/ml); median (IQR) & $52.0(33.0 ; 93.5)$ & $50.5(31.0 ; 91.0)$ & $59.0(40.0 ; 115.5)$ & 0.057 \\
\hline GGT (U/ml); median (IQR) & $80.0(38.0 ; 186.0)$ & $80.0(37.0 ; 208.0)$ & $84.0(45.5 ; 166.0)$ & 0.817 \\
\hline Albumin (mg/dl); median (IQR) & $44.0(41.0 ; 46.4)$ & $43.5(39.9 ; 46.3)$ & $45.0(43.0 ; 47.0)$ & 0.016 \\
\hline Total bilirubin (mg/dl); median (IQR) & $0.7(0.5 ; 1.0)$ & $0.7(0.5 ; 1.1)$ & $0.64(0.46 ; 0.86)$ & 0.033 \\
\hline Platelet count (G/L); median (IQR) & $221.5(180.0 ; 276.8)$ & $224.0(181.0 ; 285.0)$ & $212.0(172.0 ; 250.5)$ & 0.106 \\
\hline MELD score; median (IQR) & $7.2(6.4 ; 8.5)$ & $7.5(6.4 ; 8.5)$ & $6.9(6.4 ; 7.8)$ & 0.049 \\
\hline
\end{tabular}

Table 3 Results of non-invasive fibrosis assessment

\begin{tabular}{|c|c|c|c|c|c|}
\hline & & $\begin{array}{l}\text { All patients } \\
\quad n=392\end{array}$ & $\begin{array}{c}\text { Test cohort } \\
n=307\end{array}$ & $\begin{array}{l}\text { Validation cohort } \\
\qquad n=85\end{array}$ & $P$-value \\
\hline & Liver disease etiology & & & & \\
\hline \multirow{8}{*}{$\begin{array}{l}\text { sAxl level (ng/ml); median } \\
\text { (IQR) }\end{array}$} & Liver disease, $n=361$ & $45.7(37.2 ; 60.8)$ & $44.0(36.1 ; 59.1)$ & $53.0(42.3 ; 70.1)$ & 0.002 \\
\hline & NAFLD & $41.2(33.8 ; 52.8)$ & $40.7(33.0 ; 50.8)$ & $43.7(36.4 ; 57.5)$ & 0.076 \\
\hline & Viral hepatitis & $56.6(43.3 ; 71.7)$ & $56.5(43.0 ; 77.3)$ & $57.3(43.4 ; 70.8)$ & 0.945 \\
\hline & AIH/CLD/Overlap & $45.6(37.6 ; 70.2)$ & $43.7(36.2 ; 60.3)$ & $77.4(51.9 ; 83.0)$ & 0.001 \\
\hline & DILI & $47.9(34.5 ; 75.4)$ & $62.2(36.3 ; 103.0)$ & n.d. & n.d. \\
\hline & ALD & $63.5(47.4 ; 92.1)$ & $63.5(47.4 ; 92.1)$ & n.d. & n.d. \\
\hline & Cryptogenic & $51.2(38.7 ; 67.2)$ & $50.1(38.1 ; 62.7)$ & n.d. & n.d. \\
\hline & Healthy controls, $n=31$ & $40.2(34.2 ; 48.0)$ & $40.2(34.2 ; 48.0)$ & n.d. & n.d. \\
\hline \multirow[t]{8}{*}{ sAxl/albumin; median (IQR) } & Liver disease, $n=361$ & $(0.82 ; 1.38)$ & $1.00(0.80 ; 1.39)$ & $1.21(0.89 ; 1.56)$ & 0.004 \\
\hline & NAFLD & $0.92(0.75 ; 1.14)$ & $0.92(0.75 ; 1.13)$ & $0.93(0.80 ; 1.32)$ & 0.322 \\
\hline & Viral hepatitis & $1.29(0.97 ; 1.69)$ & $1.32(0.96 ; 1.94)$ & $1.28(0.98 ; 1.58)$ & 0.537 \\
\hline & AIH/CLD/Overlap & $1.07(0.86 ; 1.56)$ & $0.99(0.84 ; 1.40)$ & $1.94(1.20 ; 2.46)$ & 0.002 \\
\hline & DILI & $1.16(0.78 ; 1.78)$ & $1.50(0.86 ; 2.63)$ & n.d. & \\
\hline & ALD & $1.72(1.07 ; 2.59)$ & $1.72(1.07 ; 2.59)$ & n.d. & \\
\hline & Cryptogenic & $1.11(0.86 ; 1.70)$ & $1.08(0.85 ; 1.68)$ & n.d. & \\
\hline & Healthy controls, $n=31$ & $0.85(0.78 ; 1.04)$ & $0.85(0.78 ; 1.04)$ & n.d. & \\
\hline \multirow{8}{*}{$\begin{array}{l}\text { ELF }^{\text {TM }} \text { test }(\mathrm{ng} / \mathrm{ml}) ; \text { median } \\
(\mathrm{IQR})\end{array}$} & Liver disease, $n=305$ & $9.0(8.3 ; 10.1)$ & $9.2(8.4 ; 10.5)$ & $8.7(8.1 ; 9.5)$ & 0.012 \\
\hline & NAFLD, $n=162$ & $8.7(8.1 ; 9.5)$ & $8.8(8.2 ; 9.7)$ & $8.3(7.9 ; 9.0)$ & 0.009 \\
\hline & Viral hepatitis, $n=66$ & $9.3(8.6 ; 10.3)$ & $9.9(8.8 ; 11.0)$ & $9.0(8.6 ; 9.8)$ & 0.033 \\
\hline & AlH/CLD/Overlap, $n=62$ & $9.4(8.3 ; 10.8)$ & $9.4(8.3 ; 10.7)$ & $9.0(8.3 ; 11.6)$ & 0.781 \\
\hline & DILI & n.d. & n.d. & n.d. & n.d. \\
\hline & ALD, $n=13$ & $11.5(10.1 ; 12.4)$ & $11.5(10.1 ; 12.4)$ & n.d. & n.d. \\
\hline & Cryptogenic & n.d. & n.d. & n.d. & n.d. \\
\hline & Healthy controls, $n=27$ & $8.7(8.0 ; 9.3)$ & $8.7(8.0 ; 9.3)$ & n.d. & n.d. \\
\hline \multirow{8}{*}{$\begin{array}{l}\text { Fibroscan (kPa); median } \\
\text { (IQR) }\end{array}$} & Liver disease, $n=131$ & $7.9(5.8 ; 11.8)$ & $8.0(6.0 ; 11.6)$ & $7.8(5.4 ; 11.8)$ & 0.715 \\
\hline & NAFLD, $n=75$ & $8.1(6.0 ; 10.6)$ & $8.4(6.2 ; 11.4)$ & $7.6(5.8 ; 10.5)$ & 0.841 \\
\hline & Viral hepatitis, $n=33$ & $8.8(4.9 ; 12.6)$ & n.d. & $8.8(4.9 ; 12.5)$ & n.d. \\
\hline & AlH/CLD/Overlap, $n=18$ & $7.2(5.9 ; 10.1)$ & $6.4(5.8 ; 10.4)$ & $7.8(6.9 ; 10.0)$ & 0.328 \\
\hline & DILI & n.d. & n.d. & n.d. & n.d. \\
\hline & ALD & n.d. & n.d. & n.d. & n.d. \\
\hline & Cryptogenic & n.d. & n.d. & n.d. & n.d. \\
\hline & Healthy controls, $n=0$ & n.d. & n.d. & n.d. & n.d. \\
\hline
\end{tabular}

Results of non-invasive fibrosis markers were compared by Mann Whitney $U$ - test. n.d., not determined

significantly higher sAxl levels found in patients with $\mathrm{PBC}$ which was caused by a selection bias due to small sample size since only 4 patients of the validation cohort were compared to 13 patients of the test cohort. Overall sAxl levels increased according to the stage of fibrosis (Figure 1). sAxl levels were significantly higher in patients with $\mathrm{F} 4$ as compared to F0 to F3 (median sAxl: 71.6, interquartile range (IQR) [53.91; 94.73] versus 43.4, IQR [35.52; 55.71]), as well as in patients with cirrhosis according to imaging compared to patients without cirrhosis as well as healthy controls (median sAxI: 72.06, IQR [54.06; 9.01] versus 43.3, IQR [35.85; 55.54] versus 40.2 IQR [34.2; 48.0]) (Figure 1). Together, these data reveal that $\mathrm{SAxl}$ levels do not significantly change at early stages of hepatic fibrosis (F0-F3) whereas sAxl is highly elevated in liver cirrhosis (F4) compared to advanced fibrosis (F3).

Accuracy of SAxI to predict advanced liver fibrosis increases if combined with albumin. The AUC for the detection of $F \geq 2, F \geq 3$, and $F 4$ compared to patients, and cirrhosis was $0.737,0.749,0.801$, and 0.807 , respectively (Table 4). Calculating a sAxl/albumin ratio increased the AUC to $0.763,0.776,0.826$, and 0.831 , respectively (Table 4, Supplementary Figure 1). sAxl/ albumin ratios 


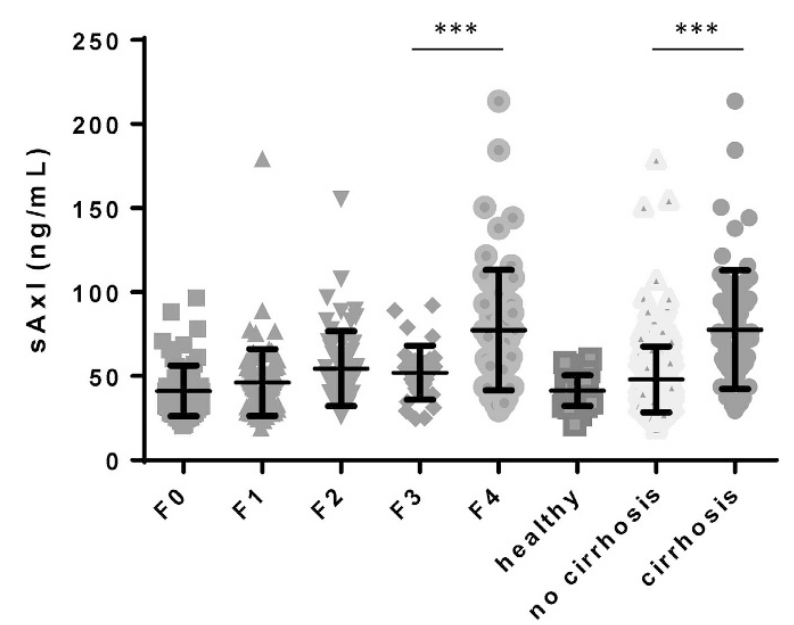

Figure 1 sAxl serum levels according to stage of fibrosis as well as presence of liver cirrhosis based on imaging methods. Statistical significant differences are expressed as asterisks: ${ }^{* * *} P<0.001$

according to fibrosis grade and cirrhosis are illustrated in Figures $2 \mathrm{a}$ and $\mathrm{d}$.

sAxI/albumin ratio has acceptable accuracy compared to ELF test and TE. ELF test and TE showed excellent diagnostic accuracy for the detection of liver fibrosis and cirrhosis. Data on their diagnostic performance are shown in Table 4 and Supplementary Figure 2. Yet, in terms of TE, $10.9 \%(n=8 / 73)$ patients of the test cohort, and $16.4 \%$ ( $n=14 / 85$ ) of the validation cohort had to be excluded due to unreliable measurements. Thus, in total 131 of 153 measurements were available for statistical analysis (Table 1).

sAxl/albumin showed an acceptable accuracy for the detection of $F \geq 2, F \geq 3, F 4$, and cirrhosis in comparison to ELF ${ }^{\mathrm{TM}}$ test (Figure 3, Table 4) as well as TE (Table 4). Spearman's correlation coefficients of $s A x l$ with sAxl/albumin ratio, ELF test, and TE were $0.969(P<0.001), 0.465$ $(P<0.001)$, and $0.215(P=0.007)$, respectively. sAxl/albumin ratio correlated with ELF ${ }^{\mathrm{TM}}$ test and TE show a Spearman's correlation coefficient of $0.526(P<0.001)$, and 0.240 $(P=0.003)$, respectively.

sAxI and sAxl/albumin ratio are independent of sex and BMI. SAxI and SAxl/albumin ratio were independent of sex (median sAxI [IQR]: male versus female: 46.1 [37.7; 58.1] versus 43.82 [35.48; 61.15], $P=0.518$; median $\mathrm{sAxl} /$ albumin [IQR]: 1.0 [0.83; 1.32] versus 1.02 [0.80; 1.50], $P=0.683$ ). Additionally, $\mathrm{sAxl}$ and $\mathrm{sAxl} /$ albumin ratio were independent of BMI category. In total, 35.7\% ( $n=140 / 392)$ of patients were normal weight (BMI $<25), 34.4 \%(n=135$ /392) of patients were overweight $(25 \leq \mathrm{BMI} \leq 30)$, and $29.8 \%(n=117 / 392)$ of patients fulfilled the WHO criteria of obesity (BMI $\geq 30) .{ }^{24}$ No significant differences were found between median sAxI [IQR] in normal weight, overweight and obese patients which was $45.0 \mathrm{ng} / \mathrm{ml}$ [37.9; 63.1], $47.1 \mathrm{ng} / \mathrm{ml}$ [38.4; 60.4], and $42.9 \mathrm{ng} / \mathrm{ml}$ [34.1; 55.1], respectively $(P=0.056)$. Median $\mathrm{sAxl} /$ albumin ratio [IQR] in normal weight, overweight and obese patients was 1.01
[0.84; 1.43], $1.08[0.85 ; 1.42]$, and $0.97[0.77 ; 1.28]$, respectively $(P=0.226)$.

\section{Discussion}

sAxl was recently identified to be an excellent biomarker for early $\mathrm{HCC}^{23,25}$ as well advanced fibrosis and liver cirrhosis. ${ }^{23}$ In the present study we aimed at investigating the diagnostic accuracy of $\mathrm{sAxl}$ as a biomarker for significant and advanced liver fibrosis in comparison to established non-invasive fibrosis markers such as ELF'M test and TE. The present study confirmed sAxl as an accurate biomarker of liver cirrhosis. Its diagnostic accuracy could be further increased by calculating a sAxl/albumin ratio achieving an AUC of 0.826 for the diagnosis of $F 4$. At a cut-off of 1.29 , a sensitivity of 77.8 and a specificity of 78.5 were reached. Notably, sAxl and sAxl/albuminratio was independent of sex and BMI.

Axl is a member of the TAM (Tyro3, Axl, Mer) subfamily of receptor tyrosine kinases and so far known to be involved in cancer development and mediation of chemoresistance. ${ }^{26}$ Besides affecting cell survival, proliferation, migration and angiogenesis, Axl additionally plays a role in the clearance of apoptotic cells, platelet aggregation and the regulation of pro-inflammatory cytokine production. ${ }^{27,28}$ After proteolytic cleavage, the extracellular domain of $A x l$ is released into the blood termed soluble Axl (sAxl). ${ }^{29}$ It has been shown to be elevated in serum or plasma in several diseases such as aortic aneurysm, heart failure, peripheral artery disease, CKD, systemic lupus erythematosus, rheumatoid arthritis, and preeclampsia. ${ }^{30-35}$ Furthermore, our group was able to show that $\mathrm{sAxl}$ is predominantly released by liver myofibroblasts that are generally associated with liver fibrosis progression. ${ }^{23}$ These findings were in line with a recent study showing that $A x l$ is involved in liver fibrosis progression in a Axl knockout mouse model proving that Axl signalling via Growth arrest-specific 6 is required for hepatic stellate cell activation. ${ }^{20}$

This is the first study to compare the diagnostic accuracy of $s A x l$ as a non-invasive biomarker of liver fibrosis with ELF $^{\mathrm{TM}}$ test and TE. The latter two are among the most intensively evaluated non-invasive tests for liver fibrosis and have been commercialized. The results of our study correspond well with previous reports on the diagnostic accuracy of ELF'T test and TE. ${ }^{2,6,8}$ However, both tests bear the disadvantage of high costs. ${ }^{19}$ Additionally, TE shows limitations in applicability since reliable results cannot be generated in up to $15.8 \%$ of patients mainly attributable to obesity, presence of ascites, or less operator experience..$^{8,36,37}$ Therefore, in our cohort in total $14.4 \%$ of TE tests had to be excluded from analysis.

In contrast, $\mathrm{sAxl}$ is a stable serum marker that can easily be measured by enzyme-linked immunoassay (ELISA) at low costs. ${ }^{38}$ Recently, we reported a diagnostic accuracy with an AUC of 0.918 (cut-off $53.89 \mathrm{ng} / \mathrm{ml}$ ) for the detection of $\mathrm{F} \geq 3$, and an AUC of 0.935 (cut-off $54.0 \mathrm{ng} / \mathrm{ml}$ ) for the detection of F4 in 190 chronic liver disease patients compared to healthy controls. ${ }^{23}$ In our present work we included 360 patients with chronic liver diseases as well as 31 healthy controls and we focused on discriminating significant to advanced fibrosis or 


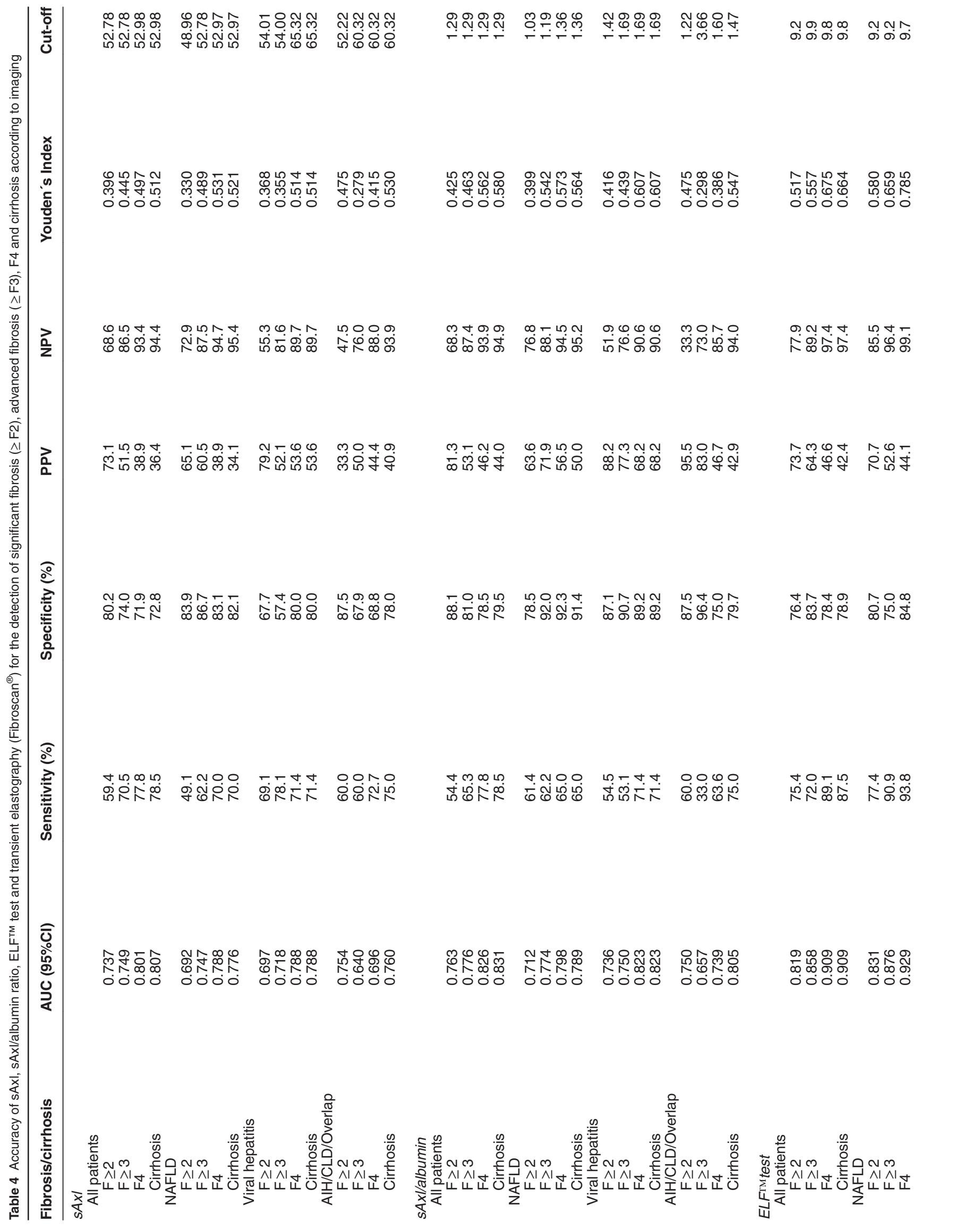




\section{6}

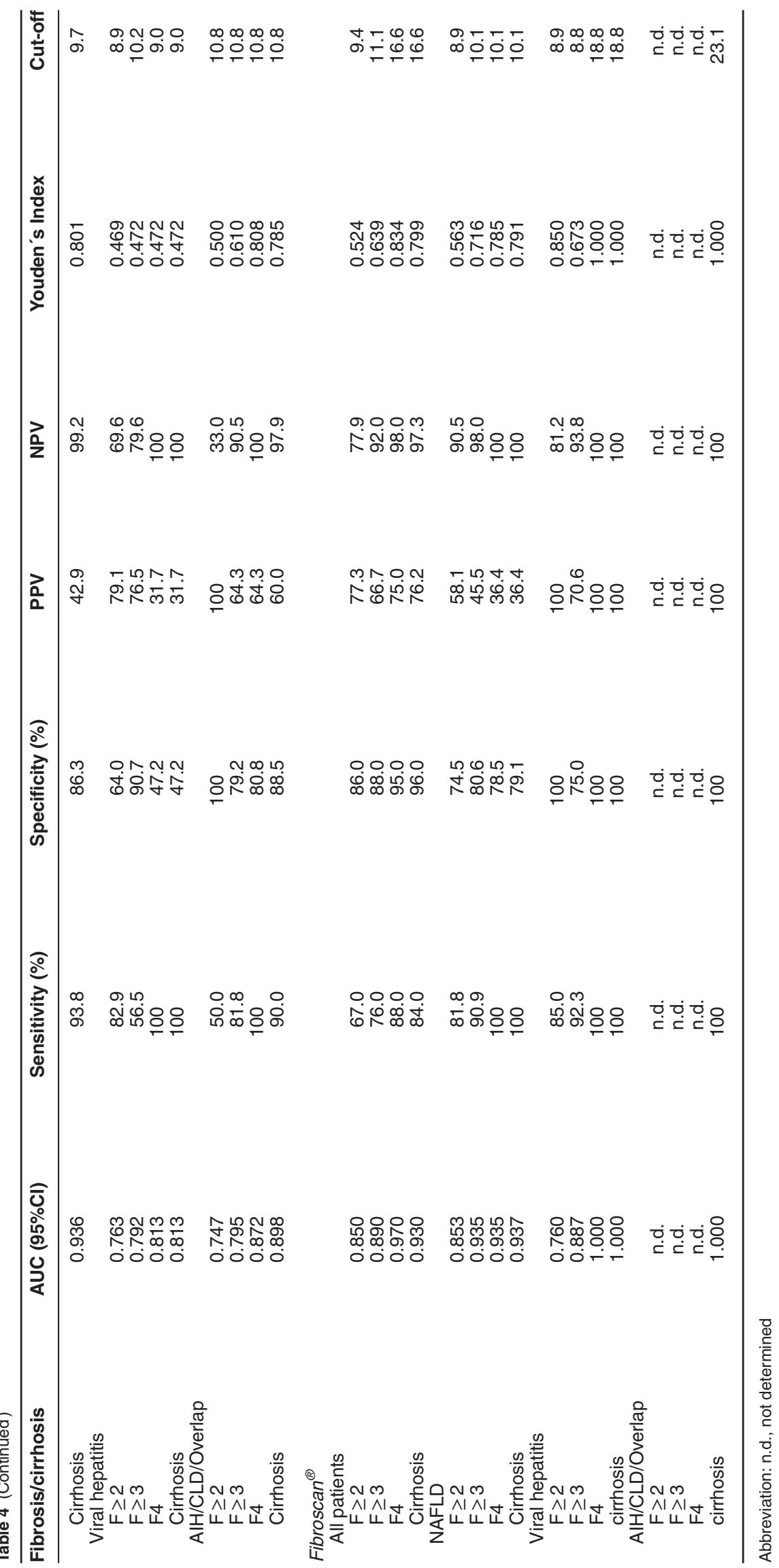


a

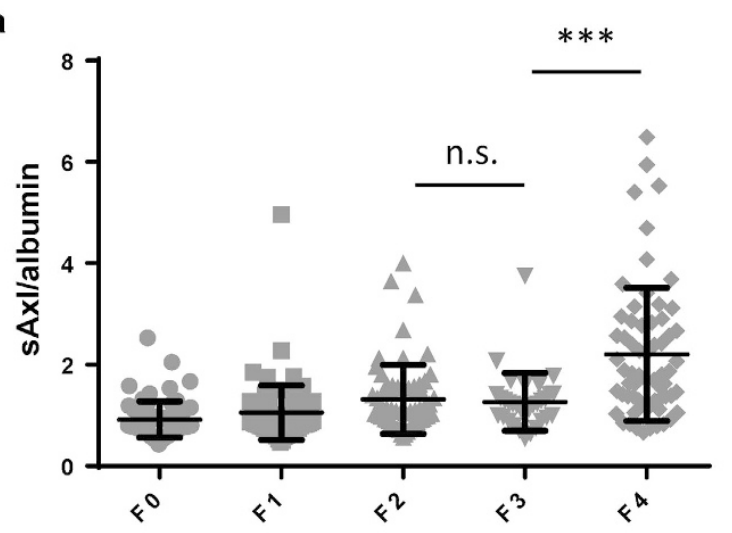

C

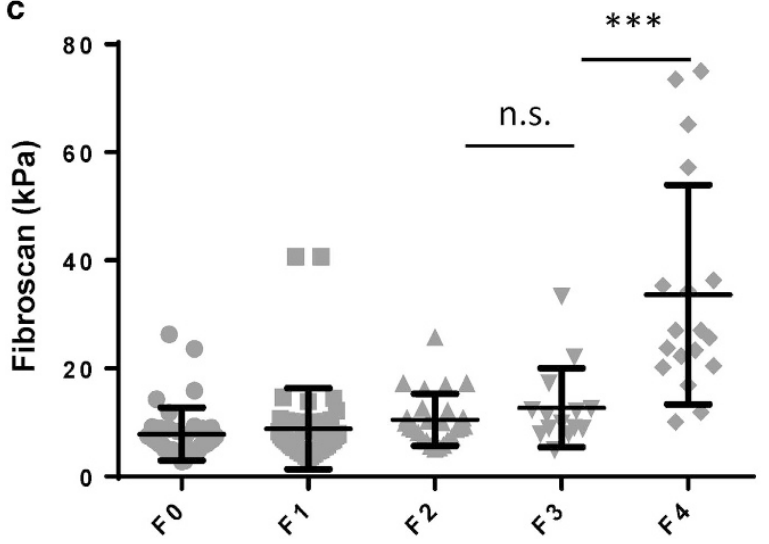

e

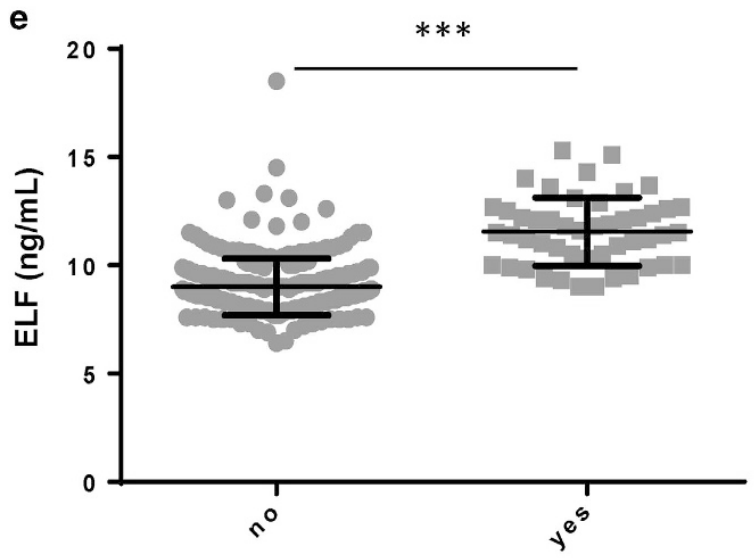

cirrhosis

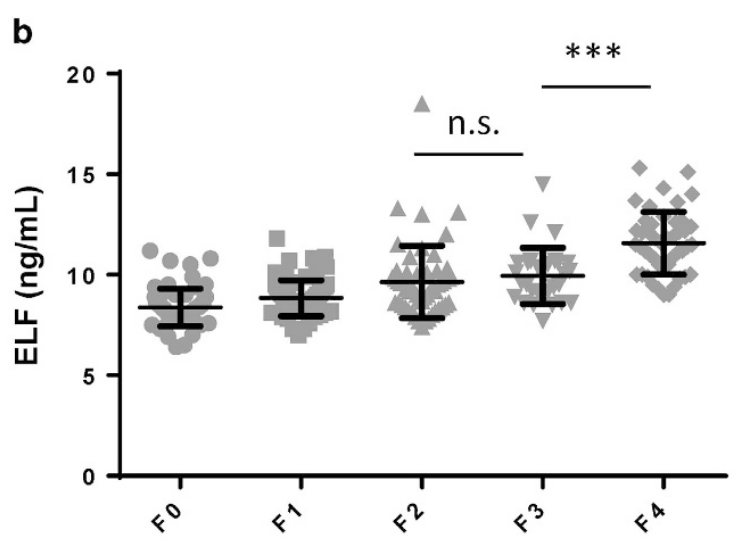

d

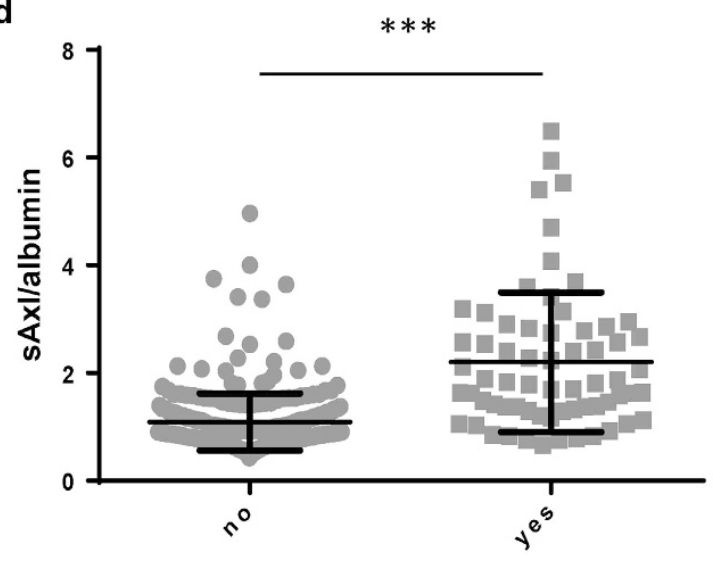

cirrhosis

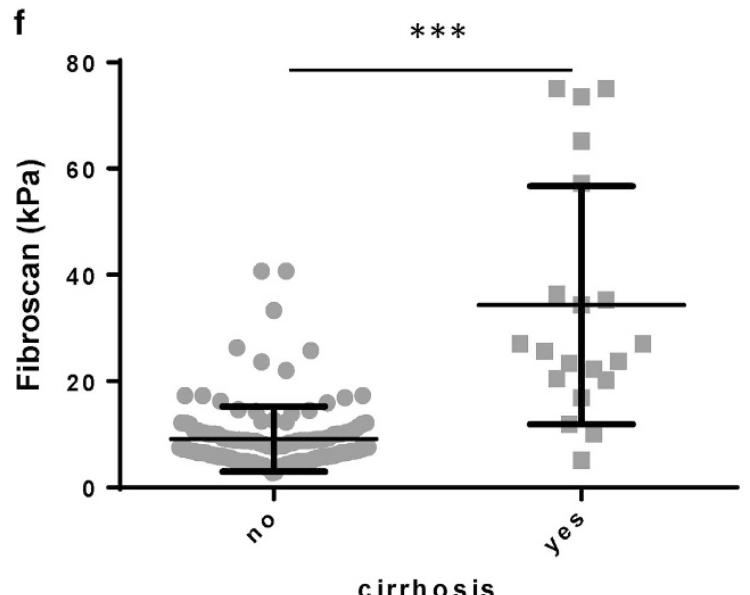

Figure 2 sAxl/albumin serum levels, ELFTM serum levels and Fibroscan results stratified according to liver biopsy (a-c) as well as stratified according to the presence of cirrhosis based on imaging test results $(\mathbf{d}-\mathbf{f})$. Statistical significant differences are expressed as asterisks: ${ }^{* * *} P<0.001$

cirrhosis from lower stages of fibrosis or healthy condition. sAxl thereby achieved an AUC of 0.801 (cut-off $52.98 \mathrm{ng} / \mathrm{ml}$ ) in identifying patients with F4. Diagnostic accuracy was increased to an AUC of 0.826 (cut-off 1.29) for the detection of F4 (reference method liver biopsy), and an AUC of 0.832 (cut-off 1.29) for the detection of liver cirrhosis (reference method imaging) by adding total serum albumin to the model. Using the same cut-off of 1.29, the AUC for the detection of significant fibrosis $(F \geq 2)$, advanced fibrosis $(F \geq 3)$, and $F 4$ was $0.763,0.776$, and 0.826 , respectively. At the cut-off of
1.29, PPV and NPV for the detection of F4 were 46.2 and $93.9 \%$, respectively, thereby reliably ruling out the presence of F4.

In this context, we have to consider that using liver biopsy as a reference method, although representing the gold standard, may automatically lead to an underestimation of non-invasive biomarkers (AUC <0.900) which is mostly due to sampling error and associated false negative results. ${ }^{39,40}$

Furthermore, liver disease etiology has been previously shown to be a major factor influencing the performance of 
a

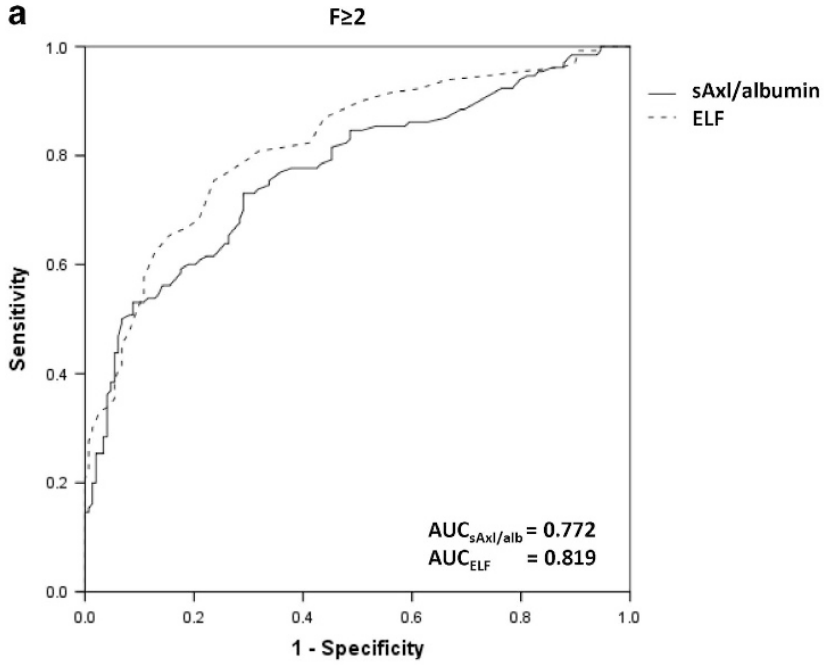

C

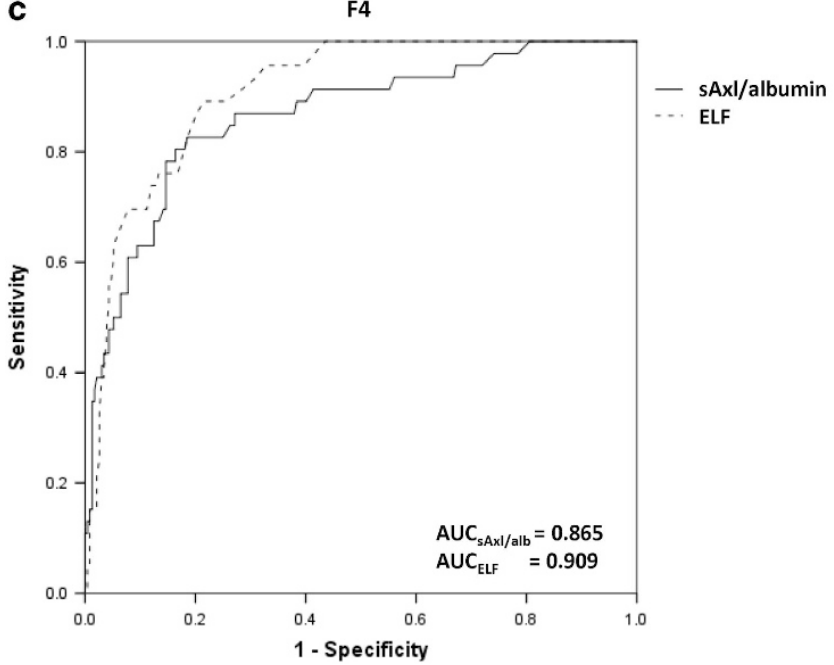

b

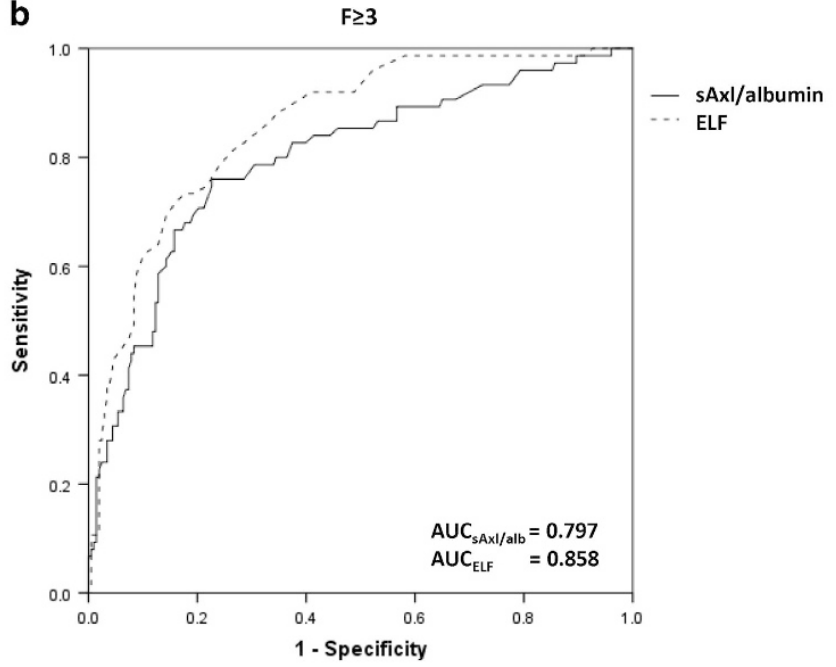

d

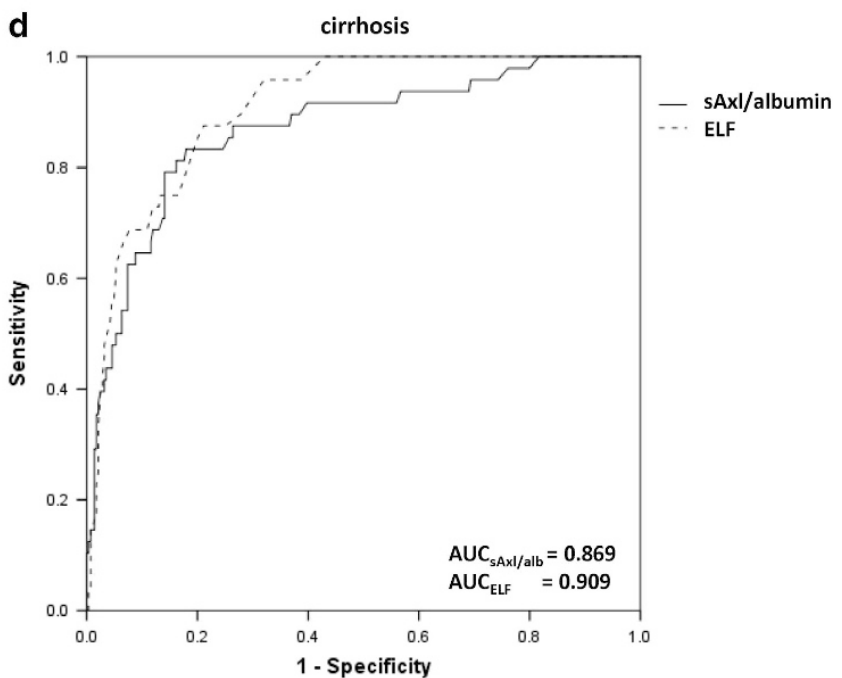

Figure 3 Comparison of the diagnostic accuracy displayed as area under the curve (AUC) of $s A x l /$ albumin and ELF test for significant fibrosis ( $F \geq 2 ;(a)$, advanced fibrosis $(\mathrm{F} \geq 3$; (b), fibrosis grade 4 ( $\mathrm{F} 4$; (c), and liver cirrhosis according to imaging (d)

non-invasive fibrosis markers. ${ }^{41}$ Especially in cholestatic liver diseases, such as PBC and PSC, data on non-invasive fibrosis assessment are limited. Additionally, classical histologic scoring systems such as those according to Ludwig, fibrosis may be only partly addressed through stages 3 and 4 (ref. 42,43). In our study sAxl/albumin ratio showed inconsistent cut-off levels within the cohort of AlH/CLD/Overlap patients with a cut-off of 3.66 for $\geq F 3$, but a cut-off of 1.6 for the diagnosis of $\mathrm{F} 4$ maybe reflecting a bias of sample size since only 4 patients in this group were classified F3 in liver histology.

Study limitations include a possible underestimation of liver fibrosis as assessed by liver histology in CLD patients, and data on ELF'M test and TE in this patient cohort is limited. Consecutively, the accuracy calculation of $\mathrm{sAxl}$ or $\mathrm{sAxl} /$ albumin might be confounded translating into underdiagnosis of significant fibrosis. Moreover, our findings cannot be uncritically translated to patients with DILI, ALD or cryptogenic liver disease due to low patient numbers in these groups.
Heart failure was excluded by the assessment of medical history and physical examination, but echocardiography was not routinely performed in all patients to exclude clinically inevitable or unknown heart failure. Therefore, sAxl levels might be increased due to unknown heart failure leading to false positive results in some patients. ${ }^{34}$ As CKD was reported to correlate with increased $\mathrm{sAxl}$ in plasma, ${ }^{35}$ we cannot exclude enhanced levels of sAxI due to mild CKD (stages 1-3) in $4.1 \%$ of our patients (15/361). However, median sAxl levels were lower in our patients with CKD than in patients without ( 48.09 versus $93.98 \mathrm{ng} / \mathrm{ml}$ ). Neither the study by Batlle et al showing increased sAxl levels in heart failure, ${ }^{34}$ nor the study be Lee and colleagues reporting elevated sAxI levels in patients with $C_{K}{ }^{35}$ excluded liver diseases in their study cohorts. Further studies are warranted to investigate the true influence of heart failure and CKD on sAxl levels in liver disease patients.

In conclusion, sAxl/albumin ratio is an accurate marker of advanced liver fibrosis and cirrhosis in NAFLD and viral 
hepatitis. Due to its easy applicability and low costs it is suitable as screening parameter for significant to advanced liver fibrosis and cirrhosis, especially in case if TE is not available or not applicable. The diagnostic accuracy of sAxl in $\mathrm{AlH}$ and cholestatic liver diseases should be confirmed in different patient cohorts.

\section{Materials and Methods}

Study population. Consecutive male and female patients with chronic liver disease as well as healthy volunteers were prospectively included in this study. Liver cirrhosis was either diagnosed by liver biopsy, liver imaging, i.e. ultrasound, computed tomography (CT) or magnetic resonance imaging (MRI), or both biopsy and imaging. Liver biopsy was evaluated by two independent pathologists $(\mathrm{CL}$ and HPD). Liver fibrosis was graded either according to Kleiner, ${ }^{44}$ Ludwig $^{42}$ or METAVIR $^{45}$ as appropriate. Blood sampling including liver function tests was performed on the day of liver biopsy or, if biopsy was not executed, within one week of liver imaging. Immediately after each blood withdrawal, one sample of whole blood was centrifuged according to a standardized protocol and stored as serum aliquots at $-80^{\circ} \mathrm{C}$ for later analysis of SAxI and ELFTM levels. In healthy volunteers liver disease was excluded by the thorough assessment of medical history, extensive laboratory tests as well as ultrasound. A test cohort was first investigated (Medical University of Vienna) followed by a validation cohort (Medical University of Graz) to confirm our findings. The medical history was thoroughly assessed in all patients and a complete physical examination was performed. Patients with acute infections, heart failure, immune-mediated diseases other than liver disease were not included in the study. Patients with CKD were categorized into CKD stages 1 to 5 (ref. 46). The study was conducted in accordance with the guidelines of the Declaration of Helsinki (1964, including current revisions) and GCP Guidelines after approval of the ethics committee of the Medical University of Vienna and Graz. All patients signed a written informed consent prior to study inclusion.

Commercialized liver fibrosis tests. ELF test was measured in serum on the ADVIA Centaur Immunoassay system according to the manufacturer's protocol (Siemens Healthcare Diagnostics Inc., NY, USA). TE using Fibroscan ${ }^{\circledR}$ (Echosens, Paris, France) was performed in fasted state and according to the manufacturer's instructions. Results showing $<10$ valid measurements, a success rate $<60 \%$ or an IQR $>30 \%$ were excluded from the analysis.

Enzyme-linked immunosorbent assay (ELISA). sAxl was measured in serum by solid phase sandwich ELISAs according to manufacturer's protocol (R\&D Systems, Minneapolis, USA) including modifications for optimization of the assay as published previously. ${ }^{23,38}$

Statistical analysis. Quantitative variables were expressed as median with interquartile range (IQR) and compared by Mann-Whitney U-tests. Qualitative variables were described using absolute and relative frequencies and were compared by Chi-Square- or Fisher's exact tests, as appropriate. Comparison of more than two groups was done by the use of the Kruskal Wallis test. The Spearman's correlation coefficient was used in order to assess correlations between all utilized non-invasive tests. The accuracy of sAxI was assessed by AUC to predict (1) significant fibrosis ( $\geq F 2$ ), (2) advanced fibrosis ( $\geq F 3$ ), (3) F4, and (4) liver cirrhosis according to imaging as $(A)$ a single marker and in combination with (C) serum albumin levels (mg/dl; expressed as sAxl/albumin ratio). In order to identify optimal cut-off values, the Youden index was calculated. ${ }^{47}$ Additionally, sensitivity, specificity, PPV, and NPV were computed. $P$-values $<0.05$ were considered statistically significant. Statistical analysis was performed using IBM Statistics SPSS 24.0 (IBM Corp., Armonk, NY).

\section{Conflict of Interest}

The authors declare no conflict of interest.

Acknowledgements. This work was supported by funds of the Oesterreichische Nationalbank (Oesterreichische Nationalbank, Anniversary Fund, project number: 16673) (to WM).

\section{Publisher's Note}

Springer Nature remains neutral with regard to jurisdictional claims in published maps and institutional affiliations.

1. European Association for the Study of the Liver. Electronic address: easloffice@easloffice. eu, EASL Recommendations on Treatment of Hepatitis C 2016. Journal of hepatology 2017; 66: 153-194.

2. European Association for the Study of the Liver (EASL); European Association for the Study of Diabetes (EASD); European Association for the Study of Obesity (EASO), EASL-EASDEASO Clinical Practice Guidelines for the management of non-alcoholic fatty liver disease. $J$ Hepatol 2016; 64: 1388-1402.

3. European Association for Study of Liver; Asociacion Latinoamericana para el Estudio de Higado, EASL-ALEH Clinical Practice Guidelines: Non-invasive tests for evaluation of liver disease severity and prognosis. J Hepatol 2015; 63: 237-264.

4. Ekstedt M, Hagstrom H, Nasr P, Fredrikson M, Stal P, Kechagias $S$ et al. Fibrosis stage is the strongest predictor for disease-specific mortality in NAFLD after up to 33 years of follow-up. Hepatology 2015; 61: 1547-1554

5. D'Amico G, Garcia-Tsao G, Pagliaro L. Natural history and prognostic indicators of survival in cirrhosis: a systematic review of 118 studies. J Hepatol 2006; 44: 217-231.

6. Chen X, Wen H, Zhang X, Dong C, Lin H, Guo Y et al. Development of a Simple Noninvasive Model to Predict Significant Fibrosis in Patients with Chronic Hepatitis B: Combination of Ultrasound Elastography, Serum Biomarkers, and Individual Characteristics. Clin Trans Gastroenterol 2017; 8: e84.

7. Angulo P, Bugianesi E, Bjornsson ES, Charatcharoenwitthaya P, Mills PR, Barrera F et al. Simple noninvasive systems predict long-term outcomes of patients with nonalcoholic fatty liver disease. Gastroenterology 2013; 145: 782-789 e784.

8. Friedrich-Rust M, Rosenberg W, Parkes J, Herrmann E, Zeuzem S, Sarrazin C. Comparison of ELF, FibroTest and FibroScan for the non-invasive assessment of liver fibrosis. BMC Gastroenterol 2010; 10: 103

9. Guha IN, Parkes J, Roderick P, Chattopadhyay D, Cross R, Harris S et al. Noninvasive markers of fibrosis in nonalcoholic fatty liver disease: Validating the European Liver Fibrosis Panel and exploring simple markers. Hepatology 2008; 47: 455-460.

10. Adler M, Gulbis B, Moreno C, Evrard S, Verset G, Golstein P et al. The predictive value of FIB-4 versus FibroTest, APRI, Fibrolndex and Forns index to noninvasively estimate fibrosis in hepatitis C and nonhepatitis C liver diseases. Hepatology 2008; 47: 762-763 author reply 763 .

11. Angulo P, Hui JM, Marchesini G, Bugianesi E, George J, Farrell GC et al. The NAFLD fibrosis score: a noninvasive system that identifies liver fibrosis in patients with NAFLD. Hepatology 2007; 45: 846-854.

12. Lok AS, Ghany MG, Goodman ZD, Wright EC, Everson GT, Sterling RK et al. Predicting cirrhosis in patients with hepatitis $\mathrm{C}$ based on standard laboratory tests: results of the HALTC cohort. Hepatology 2005; 42: 282-292.

13. Leroy V, Sturm N, Faure $P$, Trocme $C$, Marlu A, Hilleret MN et al. Prospective evaluation of FibroTest(R), FibroMeter(R), and HepaScore(R) for staging liver fibrosis in chronic hepatitis B: comparison with hepatitis C. J Hepatol 2014; 61: 28-34.

14. Ratziu V, Massard J, Charlotte F, Messous D, Imbert-Bismut F, Bonyhay L et al. Diagnostic value of biochemical markers (FibroTest-FibroSURE) for the prediction of liver fibrosis in patients with non-alcoholic fatty liver disease. BMC Gastroenterol 2006; 6: 6.

15. Mikolasevic I, Orlic L, Franjic N, Hauser G, Stimac D, Milic S. Transient elastography (FibroScan $((\mathrm{R})))$ with controlled attenuation parameter in the assessment of liver steatosis and fibrosis in patients with nonalcoholic fatty liver disease - Where do we stand? World $\mathrm{J}$ Gastroenterol 2016; 22: 7236-7251.

16. Cassinotto C, Boursier J, de Ledinghen V, Lebigot J, Lapuyade B, Cales $P$ et al. Liver stiffness in nonalcoholic fatty liver disease: A comparison of supersonic shear imaging, FibroScan, and ARFI with liver biopsy. Hepatology 2016; 63: 1817-1827.

17. Kim JH, Kim MN, Han KH, Kim SU. Clinical application of transient elastography in patients with chronic viral hepatitis receiving antiviral treatment. Liver Int 2015; 35: 1103-1115.

18. Nahon $P$, Kettaneh A, Tengher-Barna I, Ziol M, de Ledinghen V, Douvin C et al. Assessment of liver fibrosis using transient elastography in patients with alcoholic liver disease. $\mathrm{J} \mathrm{Hepatol}$ 2008; 49: 1062-1068.

19. Crossan C, Tsochatzis EA, Longworth L, Gurusamy K, Davidson B, Rodriguez-Peralvarez M et al. Cost-effectiveness of non-invasive methods for assessment and monitoring of liver fibrosis and cirrhosis in patients with chronic liver disease: systematic review and economic evaluation. Health Technol Assess 2015; 19: 1-409.

20. Barcena C, Stefanovic M, Tutusaus A, Joannas L, Menendez A, Garcia-Ruiz C et al. Gas6/ Axl pathway is activated in chronic liver disease and its targeting reduces fibrosis via hepatic stellate cell inactivation. J Hepatol 2015; 63: 670-678.

21. Uehara S, Gotoh K, Handa H, Maki Y. Plasma levels of growth arrest specific protein (Gas6) and the soluble form of its tyrosine kinase receptor $\mathrm{Axl}(\mathrm{sAxl})$ in patients with hepatocellular carcinoma. J Cancer Ther 2013; 4: 632-639.

22. Uehara S, Fukuzawa $Y$, Matuyama T, Gotoh K. Role of Tyro3, Axl and Mer receptors and their ligands (Gas6 and Protein S) in patients with hepatocellular carcinoma. J Cancer Ther 2017; 8: 112-130.

23. Dengler M, Staufer K, Huber H, Stauber R, Bantel H, Weiss $\mathrm{KH}$ et al. Soluble Axl is an accurate biomarker of cirrhosis and hepatocellular carcinoma development: results from a large scale multicenter analysis. Oncotarget 2017; 8: 46234-46248. 
24. World Health Organization. Report of a WHO consultation on obesity. Obesity: preventing and managing the global epidemic. WHO 2000; 894: 1-253.

25. Reichl P, Fang M, Starlinger P, Staufer K, Nenutil R, Muller P et al. Multicenter analysis of soluble Axl reveals diagnostic value for very early stage hepatocellular carcinoma. Int $J$ Cancer 2015; 137: 385-394.

26. Vouri M, Hafizi S. TAM Receptor Tyrosine Kinases in Cancer Drug Resistance. Cancer Res 2017; 77: 2775-2778.

27. Hafizi S, Dahlback B. Signalling and functional diversity within the Axl subfamily of receptor tyrosine kinases. Cytokine Growth Factor Rev 2006; 17: 295-304

28. Graham DK, DeRyckere D, Davies KD, Earp HS. The TAM family: phosphatidylserine sensing receptor tyrosine kinases gone awry in cancer. Nature Rev Cancer 2014; 14: 769-785.

29. O'Bryan JP, Fridell YW, Koski R, Varnum B, Liu ET. The transforming receptor tyrosine kinase, Axl, is post-translationally regulated by proteolytic cleavage. J Biol Chem 1995; 270 551-557.

30. Ekman C, Gottsater A, Lindblad B, Dahlback B. Plasma concentrations of Gas6 and soluble Axl correlate with disease and predict mortality in patients with critical limb ischemia. Clin Biochem 2010; 43: 873-876.

31. Ekman C, Site DF, Gottsater A, Lindblad B, Dahlback B. Plasma concentrations of growth arrest specific protein 6 and the soluble form of its tyrosine kinase receptor Axl as markers of large abdominal aortic aneurysms. Clin Biochem 2010; 43: 110-114.

32. Ekman C, Linder A, Akesson P, Dahlback B. Plasma concentrations of Gas6 (growth arrest specific protein 6 ) and its soluble tyrosine kinase receptor sAxl in sepsis and systemic inflammatory response syndromes. Crit care 2010; 14: R158.

33. Liu X, Gong Y, Jia J, Bai Y, Gui S, Wang T et al. Plasma concentrations of sAxl are associated with severe preeclampsia. Clin Biochem 2014; 47: 173-176.

34. Batlle M, Recarte-Pelz P, Roig E, Castel MA, Cardona M, Farrero $M$ et al. AXL receptor tyrosine kinase is increased in patients with heart failure. Int J Cardiol 2014; 173: 402-409.

35. Lee IJ, Hilliard BA, Ulas M, Yu D, Vangala C, Rao S et al. Monocyte and plasma expression of TAM ligand and receptor in renal failure: Links to unregulated immunity and chronic inflammation. Clin Immunol 2015; 158: 231-241.

36. Castera L, Foucher J, Bernard PH, Carvalho F, Allaix D, Merrouche W et al. Pitfalls of liver stiffness measurement: a 5-year prospective study of 13,369 examinations. Hepatology 2010; 51: 828-835.

37. Wong GL, Wong VW, Chim AM, Yiu KK, Chu SH, Li MK et al. Factors associated with unreliable liver stiffness measurement and its failure with transient elastography in the Chinese population. J Gastroenterol Hepatol 2011; 26: 300-305.

38. Dengler M, Huber H, Muller CJ, Zellmer A, Rauch P, Mikulits W. Accurate Determination of Soluble Axl by Enzyme-Linked Immunosorbent Assay. Assay Drug Dev Technol 2016; 14: 543-550.
39. Mehta SH, Lau B, Afdhal NH, Thomas DL. Exceeding the limits of liver histology markers. $J$ Hepatol 2009; 50: 36-41.

40. Poynard T, Halfon P, Castera L, Munteanu M, Imbert-Bismut F, Ratziu V et al Standardization of ROC curve areas for diagnostic evaluation of liver fibrosis markers based on prevalences of fibrosis stages. Clin Chem 2007; 53: 1615-1622.

41. Sebastiani G, Castera L, Halfon P, Pol S, Mangia A, Di Marco V et al. The impact of liver disease aetiology and the stages of hepatic fibrosis on the performance of non-invasive fibrosis biomarkers: an international study of 2411 cases. Aliment Pharmacol Ther 2011; 34 : 1202-1216.

42. Ludwig J, Dickson ER, McDonald GS. Staging of chronic nonsuppurative destructive cholangitis (syndrome of primary biliary cirrhosis). Virchows Arch A Pathol Anatom Histol 1978; 379: 103-112.

43. Corpechot C. Utility of Noninvasive Markers of Fibrosis in Cholestatic Liver Diseases. Clin Liver Dis 2016; 20: 143-158.

44. Kleiner DE, Brunt EM, Van Natta M, Behling C, Contos MJ, Cummings OW et al. Design and validation of a histological scoring system for nonalcoholic fatty liver disease. Hepatology 2005; 41: 1313-1321.

45. Bedossa P, Poynard T. An algorithm for the grading of activity in chronic hepatitis $C$. The METAVIR Cooperative Study Group. Hepatology 1996; 24: 289-293.

46. Isakova T, Nickolas TL, Denburg M, Yarlagadda S, Weiner DE, Gutiérrez OM. KDIGO 2017 Clinical Practice Guideline Update for the Diagnosis, Evaluation, Prevention and Treatment of Chronic Kidney Disease-Mineral and Bone Disorder (CKD-MBD). Kidney International Supplements 2017; 7 : 1-59.

47. Youden WJ. Index for rating diagnostic tests. Cancer 1950; 3: 32-35.

cc (i) Cell Death and Disease is an open-access journal published by Nature Publishing Group. This work is licensed under a Creative Commons Attribution 4.0 International License. The images or other third party material in this article are included in the article's Creative Commons license, unless indicated otherwise in the credit line; if the material is not included under the Creative Commons license, users will need to obtain permission from the license holder to reproduce the material. To view a copy of this license, visit http://creativecommons.org/licenses/by/4.0/

(C) The Author(s) 2017

Supplementary Information accompanies this paper on Cell Death and Disease website (http://www.nature.com/cddis) 